\title{
Clinical Factors Relating to Cervical Body Volume Reduction during Curative External Beam Radiation Therapy for Head and Neck Cancer
}

Ken Takeda ${ }^{1^{*}}$, Suguru Dobashi ${ }^{1}$, Shinya Komori ${ }^{1}$, Koichi Chida ${ }^{1}$, Noriyuki Kadoya ${ }^{2}$, Satoshi Kida ${ }^{2}$, Kengo Ito ${ }^{2}$, Toshiyuki Sugawara ${ }^{2}$, Masaki Kubozono ${ }^{2}$, Rei Umezawa $^{2}$, Yojiro Ishikawa ${ }^{2}$, Takaya Yamamoto ${ }^{2}$, Maiko Kozumi ${ }^{2}$, Noriyoshi Takahashi ${ }^{2}$, Yu Katagiri², Yusuke Onozato ${ }^{2}$, Takayuki Kanai ${ }^{2}$, Kiyokazu Sato ${ }^{3}$, Kazuma Kishi ${ }^{3}$, Haruo Matsushita ${ }^{2}$, Takenori Ogawa ${ }^{4}$, Yukio Katori ${ }^{4}$ and Keiichi Jingu ${ }^{2}$

${ }^{1}$ Course of Radiological Technology, Health Sciences, Tohoku University School of Medicine, Sendai, Japan

${ }^{2}$ Department of Radiation Oncology, Tohoku University School of Medicine, Sendai, Japan

${ }^{3}$ Radiation Technology, Tohoku University Hospital, Sendai, Japan

${ }^{4}$ Department of Otorhinolaryngology-Head and Neck Surgery, Tohoku University School of Medicine, Sendai, Japan

"Corresponding author: Ken Takeda, Course of Radiological Technology, Health Sciences, Tohoku University School of Medicine, Sendai, Japan, Tel: +81-22-717-7909; E-mail: ktakeda-rad@umin.net

Received date: Nov 16, 2014, Accepted date: Dec 10, 2014, Publication date: Dec 16, 2014

Copyright: (c) 2014 Takeda K, et al. This is an open-access article distributed under the terms of the Creative Commons Attribution License, which permits unrestricted use, distribution, and reproduction in any medium, provided the original author and source are credited.

\section{Abstract}

Purpose/Objectives: Substantial cervical body volume reduction (CBVR) occurs during fractionated external beam radiation therapy (EBRT) for head-and-neck cancer (HNC) and could have potential dosimetric influences. This study aims to investigate measurable clinical factors before treatment initiation correlating with CBVR during curative EBRT in HNC patients, and to determine which patients receive the great benefit from routine adaptive radiation therapy (ART).

Materials/Methods: Fifty-six patients with oropharyngeal squamous cell carcinoma (OSCC) and 67 patients with hypopharyngeal squamous cell carcinoma (HSCC) had received curative EBRT between 2006 and 2013 were enrolled. For EBRT planning, computed tomography (CT) images were acquired before EBRT initiation and between two to seven weeks after the start of EBRT for replanning in each patient. A MATLAB program was used to evaluate the CBVR rate (CBVRR) between the initial and replanning CT imaging. The following factors were assessed for correlation with CBVRR: the $\mathrm{T}$ and $\mathrm{N}$ stage, induction and concurrent chemotherapy, the initial gross tumor volume (GTV), the GTV reduction rate (GTVRR) between the initial and replanning CT imaging, the initial body weight (BW) and the BW loss rate (BWLR) during the EBRT course.

Results: In the OSCC group, the CBVRR ranged from 1.8 to $17.1 \%$ (median, $6.8 \%$ ). In the HSCC group, the CBVRR ranged from 1.2 to $23.7 \%$ (median, 6.5\%). In non-parametric univariate analysis, the N3 stage demonstrated a greater trend with the CBVRR than the N2c $\geq$ stage in the HSCC group $(p=0.023)$, whereas marginal inclination $(p=0.096)$ was found in the OSCC group. The CBVRR was substantially related to the GTVRR $(p=0.001)$ in the HSCC group.

Conclusions: The CBVRR might be related to the bulky nodal disease in the HSCC group and possibly in the OSCC group. HNC patients corresponding to these factors may have a priority to ART.

Keywords: Head and neck cancer; External beam radiation therapy; Cervical body volume; Adaptive radiation therapy

\begin{abstract}
Abbreviations:
CBVR: Cervical Body Volume Reduction; EBRT: External Beam Radiation Therapy; HNC: Head-and-Neck Cancer; ART: Adaptive Radiation Therapy; CBV: Cervical Body Volume; OSCC: Oropharyngeal Squamous Cell Carcinoma; HSCC: Hypopharyngeal Squamous Cell Carcinoma; CT: Computed Tomography; CBVRR: Cervical Body Volume Reduction Rate; GTV: Gross Tumor Volume; GTVRR: Gross Tumor Volume Reduction Rate; BW: Body Weight; BWLR: Body Weight Loss Rate; 3-D: 3-Dimensional; IMRT: Intensitymodulated Radiation Therapy; GTVR: Gross Tumor Volume Reduction; BWL: Body Weight Loss; CC: correlation coefficient
\end{abstract}

\section{Introduction}

Radiation therapy plays an essential role in the curative treatment for head-and-neck cancer (HNC) while conserving normal tissues $[1,2]$. However, the anatomical or geometric changes that frequently occur during external beam radiation therapy (EBRT) would affect the irradiated dose distribution [1-4]. As one of those changes, substantial cervical body volume reduction (CBVR) often originates during fractionated EBRT courses for HNC and has dosimetric impacts [2,5-7].

Adaptive radiation therapy (ART) can address issues such as dose variation in tumor volumes and organs at risk due to tumor shrinkage or weight loss during treatment course [8-10]. In addition, ART can improve tumor control and quality of life $[8,9,11,12]$. Furthermore, recent novel techniques support the treatment planning processes, including automated tools that are designed to limit time consumption [13-17]. Nevertheless, several publications indicated that ART requires 
Citation: Takeda K, Dobashi S, Komori S, Chida K, Kadoya N, et al. (2014) Clinical Factors Relating to Cervical Body Volume Reduction during Curative External Beam Radiation Therapy for Head and Neck Cancer. J Nucl Med Radiat Ther 6: 203. doi: $10.4172 / 2155-9619.1000203$

Page 2 of 6

intensive labor and resources [7,18-20]. Hence, it will be critical to take into account the reduction of labor and resources, probably by selecting patients appropriately that can obtain a significant benefit from ART. However, to our knowledge, there are few publications describing tangible indication criteria to determine ART priority, and it is still ambiguous whether all patients obtain a substantial advantage from routine ART [2].

According to several authors, patients with greater reduction in the cervical volume did have clinically significant benefits from adaptive strategies $[2,20]$. Surrogating the cervical body volume by measurements of the external contour, neck separation or circumference and thickness across three specified sections of the head and neck were evaluated in previous studies $[1,2,20]$. However, these measurements were not sufficiently objective. Therefore, the purpose of this study was to calculate the cervical body volume (CBV) using a more novel technique. In addition, we aimed to identify clinical factors before treatment initiation that significantly affect CBVR during definitive EBRT for HNC. Furthermore, the initial gross tumor volume (GTV), the GTV reduction rate (GTVRR) between the planning computed tomography $(\mathrm{CT})$ imaging and the replanning $\mathrm{CT}$ imaging, the initial body weight (BW) and the BW loss rate (BWLR) during the EBRT course were also assessed for relationships to CBVR reduction (CBVRR). The eventual purpose of this study was to determine which HNC patients could receive the greatest benefit from ART from the CBVR perspective, using factors identified before the start of treatment.

\section{Materials and Methods}

\section{Patients}

With the approval of the institutional research ethics board, we retrospectively reviewed clinical and image data of 56 cases of oropharyngeal squamous cell carcinoma (OSCC) and 67 cases of hypopharyngeal squamous cell carcinoma (HSCC) treated with definitive conventional 3-dimensional (3-D) conformal EBRT between November 2006 and September 2013 at the Tohoku University Hospital. The patient characteristics of the OSCC and HSCC groups are shown in Tables 1 and 2 respectively. No patients received prior surgery in this study. Six OSCC and 14 HSCC patients underwent simultaneous thoracic curative conventional EBRT for concurrent esophageal squamous cell carcinoma (Tables 1 and 2). As induction chemotherapy, four OSCC and two HSCC patients received docetaxel, cisplatin (CDDP) and 5-fluorouracil (5-FU) (TPF regimen). One course of CDDP plus 5-FU (FP regimen) and two courses of TPF regimen were delivered to one HSCC patient. Concurrent

\begin{tabular}{|l|l|}
\hline Characteristic & Value \\
\hline Age & 51 - 85 (median, 63) \\
\hline Gender & M 63, F 4 \\
\hline Stage & $14,11,17$, A 25, B 8, C 2 \\
\hline T stage & T1 9, T2 33, T3 16, T4a 8, T4b 1 \\
\hline N stage & N0 21, N1 11, N2a 2, N2b 17, N2c 6, N3 10 \\
\hline Concurrent chemotherapy & total, 61 patients \\
\hline TPF regimen & 27 \\
\hline
\end{tabular}

chemotherapy was delivered to $83.9 \%$ of OSCC patients and $91.0 \%$ of HSCC patients. The details of concurrent chemotherapy were shown in Tables 1 and 2. The details of the CDDP-based regimen group in OSCC patients were as follows: TPF regimen in 28 patients, CDDP alone in 14 patients and FP regimen in 2 patients. In the DOC-based regimen group of OSCC patients, the details were as follows: TPF regimen in 28 patients and DOC alone in 3 patients. The details of the CDDP-based regimen group in HSCC patients were as follows: TPF regimen in 27 patients, CDDP alone in 10 patients and FP regimen in 4 patients. In the DOC-based regimen group of HSCC patients, the details were as follows: TPF regimen in 27 patients, DOC alone in 18 patients and DOC plus 5-FU in one patient.

\begin{tabular}{|l|l|}
\hline Characteristic & Value \\
\hline Age & 38 - 82 (median, 65) \\
\hline Gender & M 48, F 8 \\
\hline Stage & 6,4, A 39, B 6, C 1 \\
\hline T stage & T1 7, T2 17, T3 16, T4a 12, T4b 4 \\
\hline N stage & $\begin{array}{l}\text { N0 9, N1 3, N2a 3, N2b 20, N2c 17, } \\
\text { N3 4 }\end{array}$ \\
\hline Concurrent chemotherapy & total, 47 patients \\
\hline TPF regimen & 28 \\
\hline CDDP-based regimen & 44 \\
\hline DOC-based regimen & 31 \\
\hline None & 9 \\
\hline $\begin{array}{l}\text { Simultaneous thoracic EBRT } \\
\text { concurrent ESCC }\end{array}$ & Yes 6, No 50 \\
\hline CBVRR (\%) & 1.8 - 17.1 (median, 6.8) \\
\hline GTVRR (\%) & -9.8 to 86.8 (median, 52.7) \\
\hline BWLR (\%) & -8.7 to 14.6 (median, 4.3) \\
\hline
\end{tabular}

Table 1: The patient characteristics of the oropharyngeal squamous cell carcinoma group (total, 56 patients). Abbreviation: $\mathrm{TPF}=$ Docetaxel, cisplatin and fluorouracil, $\mathrm{CDDP}=$ Cisplatin, DOC $=$ Docetaxel, $\quad \mathrm{EBRT}=$ External beam radiation therapy, ESCC=Esophageal squamous cell carcinoma, CBVRR=Cervical body volume reduction rate, GTVRR=Gross tumor volume reduction rate, BWLR=Body weight loss rate. 
Citation: Takeda K, Dobashi S, Komori S, Chida K, Kadoya N, et al. (2014) Clinical Factors Relating to Cervical Body Volume Reduction during Curative External Beam Radiation Therapy for Head and Neck Cancer. J Nucl Med Radiat Ther 6: 203. doi: $10.4172 / 2155-9619.1000203$

Page 3 of 6

\begin{tabular}{|l|l|}
\hline CDDP-based regimen & 41 \\
\hline DOC-based regimen & 46 \\
\hline S-1 & 1 \\
\hline None & 6 \\
\hline Simultaneous thoracic EBRT for concurrent ESCC & Yes 14, No 53 \\
\hline CBVRR (\%) & $1.2-23.7$ (median, 6.5) \\
\hline GTVRR (\%) & -23.6 to 88.1 (median, 55.3) \\
\hline BWLR (\%) & -4.9 to 15.2 (median, 4.2) \\
\hline
\end{tabular}

Table 2: The patient characteristics of the hypopharyngeal squamous cell carcinoma group (total, 67 patients). Abbreviations: S-1=tegafur, gimeracil and oteracil, the other abbreviations are the same as in Table 1.

All eligible patients provided written informed consent before treatment. The institutional research ethics board approved this study.

\section{Radiotherapy}

All patients underwent head-and-neck immobilization with a thermoplastic facemask. For EBRT planning in this series, CT (Light Speed QX/I or RT16, GE Medical Systems, Waukesha, WI) images were acquired twice in each patient. First CT scans were acquired before EBRT initiation for initial planning. Next CT scans was acquired for replanning and the timing of acquisition depended on the radiation oncologists in charge. The replanning CT imaging for new plan generation was done two to seven weeks after the start of EBRT (median, 3.7 weeks; range, 2.4-4.7; OSCC group)(median, 3.9 weeks; range, 2.4-7.3; HSCC group). We prepared new thermoplastic facemask to most of the patients in replanning CT scanning, except that remarkable CBVR had not occurred during EBRT course and renewal mask was not essential. The median EBRT dose at the time of the replanning CT imaging was 38 Gy (range, 26-44 Gy) in the OSCC group and 38 Gy (range, 22-48 Gy) in the HSCC group. The Eclipse treatment planning system (Varian medical Systems, Palo Alto, CA, USA) was used for all planning. The radiation oncologists determined all target volumes. The gross tumor volume (GTV) was defined as the combined gross primary and nodal disease that was related to the OSCC or the HSCC. The 3-D conformal conventional EBRT was performed using static beams of 4-MV photons from a linear accelerator (CLINAC 23EX, Varian Medical Systems, Palo Alto, California, USA). Lateral opposing fields, anterior and lateral wedged fields, or other similar field arrangements were used for the treatment of the tumor and the adjacent lymph nodes. The details of the daily dose were as follows: $2 \mathrm{~Gy}$, five times weekly throughout the EBRT course in 55 OSCC patients and 65 HSCC patients (among the 65 HSCC patients, one patient received 36 Gy with traditional 3-D conformal EBRT and another 34 Gy using intensity-modulated radiation therapy (IMRT).); 2 Gy, five times weekly until an accumulated dose of $44 \mathrm{~Gy}$ was reached and then changed to $1.5 \mathrm{~Gy}$, bid, ten times weekly until an accumulated dose of $27 \mathrm{~Gy}$ in one OSCC patient; $1.8 \mathrm{~Gy}$, five times weekly until an accumulated dose of $39.6 \mathrm{~Gy}$ was reached and then changed to $2 \mathrm{~Gy}$, five times weekly until an accumulated dose of $30 \mathrm{~Gy}$ in one HSCC patient; $1.2 \mathrm{~Gy}$, bid, and 10 times weekly throughout the whole EBRT course in another HSCC patient. The median total EBRT dose was $70 \mathrm{~Gy}$ (range, 56-71 Gy) in OSCC patients and $70 \mathrm{~Gy}$ (range, 60-72 Gy) in HSCC patients.

\section{Cervical body volume reduction, GTV reduction and body weight loss}

Cervical body volume between the spinous process of the $\mathrm{C} 1$ and C7 level were calculated using a MATLAB (Mathworks, Natick, MA) program in both the initial and replanning CT images. The values of CBVRR were obtained from those imaging sets. The volume was determined by the image processing analysis. We first set the threshold on the CT image to exclude the treatment couch and the immobilization device of head and neck, and then converted the CT image to a binary image based on the threshold. The cervical body volume was determined from the number of voxels within the cervical body contour multiplied by the volume of a voxel obtained from the pixel spacing (approximately $1 \mathrm{~mm}$ ) and the slice thickness $(5 \mathrm{~mm})$ of the CT image. Moreover, the GTVRR was calculated by the following equation: GTVRR $=[(G T V$ on the initial planning CT imaging $)-(G T V$ on the replanning CT imaging)/(GTV on the initial-planning CT imaging)]. The body weight of each patient was measured at both before the EBRT initiation and during the EBRT course.

\section{Statistical analysis}

To explore clinical factors with respect to CBVRR, the MannWhitney $U$ test, the Kruskal-Wallis test and the Spearman rank correlation (two-tailed) were performed for non-parametric univariate analyses. A two-sided probability value of 0.05 was considered statistically significant. The following clinical variables were assessed for correlation with CBVRR: the $\mathrm{T}$ stage, the $\mathrm{N}$ stage, induction and concurrent chemotherapy, initial GTV, GTVRR, initial BW and BWLR. All statistical analyses were performed using a commercial software package (SPSS 20.0 for windows, Chicago, IL, USA).

\section{Results}

\section{Details about reduction of cervical body volume, GTV and body weight loss rate}

In the OSCC group, the CBV on the initial planning CT imaging ranged from 967.6 to $2229.5 \mathrm{~mL}$ (median, $1530.4 \mathrm{~mL}$ ), whereas the $\mathrm{CBV}$ on the replanning CT imaging ranged from 938.9 to $2077.2 \mathrm{~mL}$ (median, $1407.1 \mathrm{~mL}$ ). The CBVR ranged from 23.9 to $287.1 \mathrm{~mL}$ (median, $101.1 \mathrm{~mL}$ ). In the HSCC group, the CBV on the initial planning CT imaging ranged from 964.2 to $2294.7 \mathrm{~mL}$ (median, 1429.2 $\mathrm{mL}$ ), whereas the $\mathrm{CBV}$ on the replanning $\mathrm{CT}$ imaging ranged from 
Citation: Takeda K, Dobashi S, Komori S, Chida K, Kadoya N, et al. (2014) Clinical Factors Relating to Cervical Body Volume Reduction during Curative External Beam Radiation Therapy for Head and Neck Cancer. J Nucl Med Radiat Ther 6: 203. doi: $10.4172 / 2155-9619.1000203$

Page 4 of 6

845.1 to $2149.9 \mathrm{~mL}$ (median, $1354.2 \mathrm{~mL}$ ). The CBVR ranged from 14.6 to $349.0 \mathrm{~mL}$ (median, $103.1 \mathrm{~mL}$ ). In the OSCC group, the GTV on the initial planning CT imaging ranged from 2.7 to $211.1 \mathrm{~mL}$ (median, $46.2 \mathrm{~mL}$ ), whereas the GTV on the replanning CT imaging ranged from 1.3 to $194.9 \mathrm{~mL}$ (median, $18.6 \mathrm{~mL}$ ). The GTV reduction (GTVR) ranged from -11.1 to $153.2 \mathrm{~mL}$ (median, $17.8 \mathrm{~mL}$ ). In the HSCC group, the GTV on the initial planning CT imaging ranged from 2.0 to 429.1 $\mathrm{mL}$ (median, $25.0 \mathrm{~mL}$ ), whereas the GTV on the replanning CT imaging ranged from 0.9 to $245.5 \mathrm{~mL}$ (median, $10.1 \mathrm{~mL}$ ). The GTVR ranged from -16.0 to $222.6 \mathrm{~mL}$ (median, $11.1 \mathrm{~mL}$ ). In the OSCC group, the body weight (BW) before EBRT initiation ranged from 35.7 to 80.9 $\mathrm{kg}$ (median, $57.3 \mathrm{~kg}$ ), whereas the BW during the EBRT course ranged from 33.0 to $76.5 \mathrm{~kg}$ (median, $54.8 \mathrm{~kg}$ ). The BW loss (BWL) ranged from -4.8 to $9.5 \mathrm{~kg}$ (median, $2.4 \mathrm{~kg}$ ). In the HSCC group, the BW before EBRT initiation ranged from 37.7 to $88.3 \mathrm{~kg}$ (median, $53.5 \mathrm{~kg}$ ), whereas the BW during the EBRT course ranged from 35.6 to $80.4 \mathrm{~kg}$ (median, $52.3 \mathrm{~kg}$ ). The BWL ranged from -2.0 to $9.0 \mathrm{~kg}$ (median, 2.2 $\mathrm{kg})$.

The details of the CBVRR, GTVRR and BWLR in the OSCC and HSCC groups are listed in Tables 1 and 2 respectively.

\section{Clinical factors with respect to the cervical body volume reduction rate}

As displayed in Table 3, the N3 stage in the HSCC group was statistically related to the CBVRR $(\mathrm{p}=0.023)$. In the OSCC group, the $\mathrm{N} 3$ stage was not statistically related, but peripherally correlated $(\mathrm{p}=0.096)$. The median and range of the CBVRR with respect to the $\mathrm{N} 2 \mathrm{c} \geq$ and $\mathrm{N} 3$ stages were $6.7 \%(1.8-17.1 \%)$ and $11.3 \%(5.3-14.5 \%)$ in the OSCC group and $5.9 \%(1.2-17.6 \%)$ and $11.8 \%(1.2-23.8 \%)$ in the HSCC group, respectively. Thus, the N3 stage patients showed a greater tendency of $\mathrm{CBVR}$ than the $\mathrm{N} 2 \mathrm{c} \geq$ stage patients during the current EBRT.

\begin{tabular}{|l|l|l|}
\hline & OSCC & HSCC \\
\hline Factors & $\begin{array}{l}\text { UA } \\
\text { p value }\end{array}$ & $\begin{array}{l}\text { UA } \\
\text { p value }\end{array}$ \\
\hline T stage T1 vs. T2-4b & 0.96 & 0.94 \\
\hline T1-2 vs. T3-4b & 0.18 & 0.36 \\
\hline T1-3 vs. T4a-b & 0.073 & 0.53 \\
\hline N stage N0 vs. N1-3 & 0.87 & 0.51 \\
\hline N0-1 vs. N2a-3 & 0.52 & 0.48 \\
\hline N0-2a vs. N2b-3 & 0.78 & 0.39 \\
\hline N0-2b vs. N2c-3 & 0.61 & 0.19 \\
\hline N0-2c vs. N3 & 0.096 & $0.023^{*}$ \\
\hline Induction chemotherapy (Yes vs. No) & 1.00 & 0.326 \\
\hline Concurrent chemotherapy (Yes vs. No) & 0.704 & 0.676 \\
\hline regimen with TPF/regimen without TPF/none & 0.92 & 0.11 \\
\hline $\begin{array}{l}\text { regimen with CDDP/regimen without CDDP/ } \\
\text { none }\end{array}$ & 0.89 & 0.055 \\
\hline $\begin{array}{l}\text { regimen with DOC/regimen without DOC/ } \\
\text { none }\end{array}$ & 0.93 & 0.818 \\
\hline
\end{tabular}

\begin{tabular}{|l|l|l|}
\hline $\begin{array}{l}\text { Simultaneous thoracic EBRT for concurrent } \\
\text { EC }\end{array}$ & 0.29 & 0.15 \\
\hline Initial GTV & 0.11 & 0.18 \\
\hline GTVRR & 0.34 & $0.001^{*}$ \\
\hline Initial BW & 0.25 & 0.50 \\
\hline BWLR & 0.66 & 0.034 \\
\hline
\end{tabular}

Table 3: The correlation between the cervical body volume reduction rate and clinical factors. Abbreviations: OSCC $=$ oropharyngeal squamous cell carcinoma, HSCC = hypopharyngeal squamous cell carcinoma. GTV = Gross tumor volume, BW = Body weight. The other abbreviations are the same as in Table 1 . Note: ${ }^{*}=$ statistical significance (the correlation coefficient (CC) in the Spearman rank correlation was 0.404 ), = low statistical significance (the CC was 0.26 ).

Concurrent chemotherapy using a CDDP-based regimen showed marginal relation to CBVR in the HSCC group (Table 3). However, the median and range of CBVRR on that regimen were as follows: $8.5 \%(1.7-23.7 \%)$ in the CDDP-based concurrent chemotherapy group, $5.2 \%(1.2-15.4 \%)$ in the concurrent chemotherapy using other menus without the CDDP-based regimen group, and $8.1 \%(1.2-$ $15.4 \%$ ) in the group without any concurrent chemotherapy. Thus, there were no significant differences of the detailed CBVRR among these categories. Therefore, whereas concurrent CDDP-based chemotherapy showed a boundary trend of CBVR in the HSCC group, it was difficult to clarify a relationship between CDDP-based concurrent chemotherapy and CBVR. Similarly, the presence of induction chemotherapy was not significant in either the OSCC or the HSCC group.

As shown in Table 3, the CBVRR was significantly correlated with the GTVRR (the correlation coefficient (CC) was 0.404), whereas it did not show a substantial relation to the BWLR (the CC was 0.26 ) in the HSCC group. In the OSCC group, the CBVRR did not demonstrate a significant correlation with either the GTVRR or the BWLR.

\section{Discussion}

This retrospective study demonstrated substantial variation in the extent of the CBVR during fractionated EBRT for OSCC and HSCC. In addition, as cognizable clinical information before treatment initiation, we suggested a clinical factor relating to CBVR.

Previous publications describe that ART can improve treatment outcomes and quality of life by providing dosimetric benefits [8-12]. Nevertheless, several authors mentioned not only that ART may increase the workload for clinical staff, dosimetrists and radiation oncologists but also that there is a remarkable financial burden on the treating institution because of the increased cost of reimaging and replanning $[7,18-20]$. Therefore, we believe that it is essential to preferentially select patients who should undergo ART.

Changes of the cervical body volume often occur during fractionated EBRT courses for HNC [1,2,5-7,20]. In addition, substantial CBVR cannot be ignored as it would have dosimetric impacts on the target volume and normal tissue if the recent high conformal EBRT techniques, such as IMRT, were leveraged without adequate adaptive strategies [2,20]. According to Lai et al. [20], adaptive planning should be considered for those who have a large reduction ratio in the circumference and thickness over the level of the 
Citation: Takeda K, Dobashi S, Komori S, Chida K, Kadoya N, et al. (2014) Clinical Factors Relating to Cervical Body Volume Reduction during Curative External Beam Radiation Therapy for Head and Neck Cancer. J Nucl Med Radiat Ther 6: 203. doi: $10.4172 / 2155-9619.1000203$

Page 5 of 6

mastoid tip during the treatment. Capelle et al. [2] also described that significant reduction in neck separation during treatment would cause a reduction in the adequacy of immobilization. Hence, the adequacy of immobilization, including setup technique, due to cervical volume change may be a more pressing reason to use ART in HNC patients $[2,20]$. Therefore, we believe that clarifying clinical information relating to the CBVR is important for selecting preferential patients who should be treated with ART, including the adaptive setup technique.

As a surrogate of whole CBV measuring, previous authors assessed one- or two-dimensional measurement of CBV on a cross-sectional image. For example, Barker et al. [1] estimated the external skin contours. According to Capelle et al. [2], patients with greater reductions in neck separation did have clinically significant benefits and should be targeted for adaptive strategies. Lai et al. [20] adopted certain specified sections of the head and neck. These previous assessment of CBV appeared to be less direct methods of surveying. Hence, we utilized a MATLAB program to quantify more detailed CBV values and to avoid intra- and inter-observer variation in measurement as much as possible. Consequently, it became clear that a remarkable extent of CBVR existed. Simultaneously, we explored knowable clinical factors before treatment initiation with respect to the CBVR. As a result, advanced regional lymph node diseases such as the $\mathrm{N} 3$ stage displayed a greater inclination of CBVR than the N2c $\geq$ stage during EBRT in this study. According to Barker et al. [1],the volume loss in primary tumors and lymph nodes was larger for large tumors/ nodes and the rate of volume loss correlated highly with the initial volume of gross disease. They also indicated that the initial volume is a good determinant for the rate of volume loss during the course of radiotherapy. Similarly, in this study, one of the reasons why a greater $\mathrm{N}$ stage predicts a higher CBVR value may be because the magnitude of shrinkage in the lesser $\mathrm{N}$ stage is physically limited compared to that in the higher $\mathrm{N}$ stage. Consequently, the volume reduction in regional bulky advanced lymph node diseases might contribute to substantial CBVR values.

In our findings, the CBVRR was significantly correlated with the GTVRR in the HSCC group, but not in the OSCC group. However, the consequence should be interpreted prudently. As for mechanism of the CBVR during fractionated EBRT courses, Barker et al. [1] denoted that not only the GTVR or BWL but also factors such as fluid shifts/ edema, inflammation, or other phenomenon might be involved. The latter reasons might be dominant in the current OSCC group, however, it would be difficult to elucidate in detail. Moreover, in general, the extent of these factors would be hard to known before treatment initiation. Moreover, it should be noted that the variation in contouring the GTV among radiation oncologists would be relatively substantial, especially in small primary tumors or nodal lesions, as Barker et al. indicated [1]. There were few attempts to reduce bias of contouring GTV in this study. Therefore, this result needs to be carefully examined.

Concurrent chemotherapy using a CDDP-based regimen also displayed a boundary relationship to the CBVRR in the HSCC group. The use of CDDP-based concurrent EBRT in HNC patients is based on the report of the meta-analysis of the MACH-NC collaborative group and its subsequent update on the effects of the addition of chemotherapy to radiotherapy $[21,22]$. One plausible reason is that the concurrent CDDP-based regimen with EBRT might contribute to the CBVR by shrinking the tumor and/or nodal disease in the HSCC group. However, there were no significant disparities of the detailed
CBVRR among these categories in the HSCC. Further investigation would be needed to assert a significant relationship between CDDPbased concurrent chemotherapy and CBVR.

Several limitations exist in this retrospective study. First, there might be inter-radiation oncologist variation in the GTV delineation, as previously described. Although Yang et al. [23] made attempts to reduce the deviation of calculated values for any volume, we did not take this into account. In addition, the GTV on the replanning CT imaging were calculated to be larger than the GTV on the initial planning CT imaging in two OSCC and three HSCC patients. This may have been due to inflammatory and/or edematous change that would cause ambiguity in contouring the GTV. Another reason might be that the timing of the replanning CT imaging was uneven in this series. Hence, our estimation of the GTV might have more bias than previous reports. Moreover, we could not deny a concern that the variation in timing of replanning CT might have affected measurement of the CBVRR. Second, we did not estimate actual dosimetric changes caused by the CBVR during EBRT. Several publications reported that anatomical changes during the treatment of HNC patients would have an impact on the dose distribution and induce a loss of dose coverage to target volumes and an overdosage to normal structures $[3,9,24,25]$. Olteanu et al. [26] compared the dosimetric effects of adaptive and non-adaptive dose-painted radiotherapy and revealed that ART readjusted dose-painting by increasing the minimum and decreasing the maximum doses in the target volumes and improving the dose/volume metrics of the organ at risk. Third, this study did not include HNC patients treated with IMRT for the whole radiotherapy course. Contrary to current conventional EBRT, a steeper dose gradient, especially at the edge close to both the tumor and the normal tissue, exists in IMRT. There is a suspicion whether this result is also valid in IMRT. Therefore, prospective verification is essential to confirm this outcome. Finally, this retrospective study included a heterogeneous patient population. It was difficult to exclude a possibility that this varied population might have influenced to the outcome.

However, it is still challenging to select HNC patients who can significantly obtain benefit from ART. Olteanu et al. [26] compared the dosimetrical effects of adaptive and non-adaptive dose-painted radiotherapy and revealed considerable heterogeneity in the patientspecific benefits from the adaptive strategy. Further investigation is in progress to elucidate the predictive factors relating to the BWL or the GTVR to preferentially identify patients that should be treated with ART.

\section{Conclusions}

In this investigation, we quantified the CBVR for 56 OSCC and 67 HSCC patients who underwent fractionated EBRT. Significant variation in the CBVR was observed. The knowable clinical factors before treatment initiation associated with the CBVR include bulky regional nodal disease. As a whole strategy, HNC patients with this factor may be considered as ideal ART candidates, prior to treatment initiation. Our findings may provide a potential benefit in the appropriate selection of patients.

\section{Acknowledgements}

This work was supported by grants from the Japanese Society for Radiation Oncology 2013-14. We presented some parts of this study at the American Society for Radiation Oncology 2014 annual meeting. 
Citation: Takeda K, Dobashi S, Komori S, Chida K, Kadoya N, et al. (2014) Clinical Factors Relating to Cervical Body Volume Reduction during Curative External Beam Radiation Therapy for Head and Neck Cancer. J Nucl Med Radiat Ther 6: 203. doi: $10.4172 / 2155-9619.1000203$

Page 6 of 6

\section{References}

1. Barker JL Jr, Garden AS, Ang KK, O'Daniel JC, Wang H, et al. (2004) Quantification of volumetric and geometric changes occurring during fractionated radiotherapy for head-and-neck cancer using an integrated CT/linear accelerator system. Int J Radiat Oncol Biol Phys 59: 960-970.

2. Capelle L, Mackenzie M, Field C, Parliament M, Ghosh S, et al. (2012) Adaptive radiotherapy using helical tomotherapy for head and neck cancer in definitive and postoperative settings: initial results. Clin Oncol (R Coll Radiol) 24: 208-215.

3. Beltran M, Ramos M, Rovira JJ, Perez-Hoyos S, Sancho M, et al. (2012) Dose variations in tumor volumes and organs at risk during IMRT for head-and-neck cancer. J Appl Clin Med Phys 13: 3723.

4. Bhide SA, Davies M, Burke K, McNair HA, Hansen V, et al. (2010) Weekly volume and dosimetric changes during chemoradiotherapy with intensity-modulated radiation therapy for head and neck cancer: a prospective observational study. Int J Radiat Oncol Biol Phys 76: 1360-1368.

5. Chao M, Xie Y, Moros EG, Le QT, Xing L (2010) Image-based modeling of tumor shrinkage in head and neck radiation therapy. Med Phys 37: 2351-2358.

6. Bando R, Ikushima H, Kawanaka T, Kudo T, Sasaki M, et al. (2013) Changes of tumor and normal structures of the neck during radiation therapy for head and neck cancer requires adaptive strategy. J Med Invest 60: 46-51

7. Hansen EK, Bucci MK, Quivey JM, Weinberg V, Xia P (2006) Repeat CT imaging and replanning during the course of IMRT for head-and-neck cancer. Int J Radiat Oncol Biol Phys 64: 355-362.

8. Chen AM, Daly ME, Cui J, Mathai M, Benedict S, et al. (2014) Clinical outcomes among patients with head and neck cancer treated by intensitymodulated radiotherapy with and without adaptive replanning. Head Neck 36: 1541-1546.

9. Schwartz DL, Garden AS, Shah SJ, Chronowski G, Sejpal S, et al. (2013) Adaptive radiotherapy for head and neck cancer--dosimetric results from a prospective clinical trial. Radiother Oncol 106: 80-84.

10. Castadot P, Lee JA, Geets X, Grégoire V (2010) Adaptive radiotherapy of head and neck cancer. Semin Radiat Oncol 20: 84-93.

11. Yang H, Hu W, Wang W, Chen P, Ding W, et al. (2013) Replanning during intensity modulated radiation therapy improved quality of life in patients with nasopharyngeal carcinoma. Int J Radiat Oncol Biol Phys 85: e47-54.

12. Zhao L, Wan Q, Zhou Y, Deng X, Xie C, et al. (2011) The role of replanning in fractionated intensity modulated radiotherapy for nasopharyngeal carcinoma. Radiother Oncol 98: 23-27.

13. Li N, Zarepisheh M, Uribe-Sanchez A, Moore K, Tian Z, et al. (2013) Automatic treatment plan re-optimization for adaptive radiotherapy guided with the initial plan DVHs. Phys Med Biol 58: 8725-8738.
14. Hatt M, Cheze le Rest C, Descourt P, Dekker A, De Ruysscher D, et al. (2010) Accurate automatic delineation of heterogeneous functional volumes in positron emission tomography for oncology applications. Int J Radiat Oncol Biol Phys 77: 301-308.

15. Yang J, Amini A, Williamson R, Zhang L, Zhang Y, et al. (2013) Automatic contouring of brachial plexus using a multi-atlas approach for lung cancer radiotherapy. Pract Radiat Oncol 3: e139-e147.

16. Hofheinz F, Langner J, Petr J, Beuthien-Baumann B, Steinbach J, et al. (2013) An automatic method for accurate volume delineation of heterogeneous tumors in PET. Med Phys 40: 082503.

17. Gubbi J, Kanakatte A, Tomas K, Binns D, Srinivasan B, et al. (2011) Automatic tumour volume delineation in respiratory-gated PET images. J Med Imaging Radiat Oncol 55: 65-76.

18. Schwartz DL, Dong L (2011) Adaptive radiation therapy for head and neck cancer-can an old goal evolve into a new standard? J Oncol 2011

19. Schwartz DL1 (2012) Current progress in adaptive radiation therapy for head and neck cancer. Curr Oncol Rep 14: 139-147.

20. Lai YL, Yang SN, Liang JA, Wang YC, Yu CY, et al. (2014) Impact of body-mass factors on setup displacement in patients with head and neck cancer treated with radiotherapy using daily on-line image guidance. Radiat Oncol 9: 19.

21. Pignon JP, Bourhis J, Domenge C, DesignÃ® L. (2000) Chemotherapy added to locoregional treatment for head and neck squamous-cell carcinoma: three meta-analyses of updated individual data. MACH-NC Collaborative Group. Meta-Analysis of Chemotherapy on Head and Neck Cancer. Lancet 355: 949-955.

22. Pignon JP, le Maître A, Bourhis J; MACH-NC Collaborative Group (2007) Meta-Analyses of Chemotherapy in Head and Neck Cancer (MACH-NC): an update. Int J Radiat Oncol Biol Phys 69: S112-114.

23. Yang SN, Liao CY, Chen SW, Liang JA, Tsai MH, et al. (2011) Clinical implications of the tumor volume reduction rate in head-and-neck cancer during definitive intensity-modulated radiotherapy for organ preservation. Int J Radiat Oncol Biol Phys 79: 1096-1103.

24. Castadot P, Geets X, Lee JA, Grégoire V (2011) Adaptive functional image-guided IMRT in pharyngo-laryngeal squamous cell carcinoma: is the gain in dose distribution worth the effort? Radiother Oncol 101: 343-350.

25. Wu Q, Chi Y, Chen PY, Krauss DJ, Yan D, et al. (2009) Adaptive replanning strategies accounting for shrinkage in head and neck IMRT. Int J Radiat Oncol Biol Phys 75: 924-932.

26. Olteanu LA, Berwouts D, Madani I, De Gersem W, Vercauteren T, et al. (2014) Comparative dosimetry of three-phase adaptive and non-adaptive dose-painting IMRT for head-and-neck cancer. Radiother Oncol 111: 348-353.
This article was originally published in a special issue, entitled: "Cancer Radiation Therapy", Edited by University of Arkansas for Medical Sciences, USA 